\title{
Numerical Solution of Systems of Partial Differential Equation Using New Variational Homotopy Perturbation Method
}

\author{
O. E. Abolarin ${ }^{1}$, O. A. TAIWO ${ }^{2}$ \\ Department of Industrial Mathematics Landmark University, Omu-Aran, Nigeria. \\ Department of Mathematics University of Ilorin, Ilorin, Nigeria
}

\begin{abstract}
This research paper deals with the systems of partial differential equations by using the New Variational Homotopy Perturbation Method. The New Method does not require discritization, linearization or any restrictive assumption of any form in providing analytical or approximate solutions to linear and nonlinear equation. Theses virtues make it to be reliable and its efficiency is demonstrated with numerical examples.

Mathematics subject classification: 35k15, 35C05, 65D99, $65 M 99$

Keywords: systems of partial differential equations; Variational Iteration Method, Homotopy Perturbation Method, New Variational Homotopy Perturbation Method, Boundary Value Problems; Initial Value Problems.
\end{abstract}

\section{Introduction}

System of partial differential equations have attracted much attention in a variety of applied Sciences. The general ideas and the essential features of these systems are of wide applicability. These system were formally derived to describe wave propagation, to control the shallow water waves and to examine the chemical reaction-diffusion model of Brusselator. The method of characteristics, the Riemann invariants, Adomian decomposition method [1-3], Homotopy perturbation method [4-9], Homotopy analysis method and Laplace decomposition method [10-17], were the commonly used methods. In order to improve on existing method of solution we introduce the New Variational Homotopy Perturbation Method for System of partial differential equations which is time cost effective and users friendly.

\section{New Variational Homotopy Perturbation Method}

We extent the new scheme to systems of partial differential equations of form:

$$
\begin{aligned}
& L_{1} U(x, t)+N_{1}(U(x, t), V(x, t))=f(x, t) \\
& L_{2} U(x, t)+N_{2}(U(x, t), V(x, t))=g(x, t)
\end{aligned}
$$

Where $\mathrm{L}_{1}$ and $\mathrm{L}_{2}$ are linear differential operators, with respect to time; $\mathrm{N}_{1}$ and $\mathrm{N}_{2}$ are non-linear operators and $\mathrm{f}(\mathrm{x}, \mathrm{t})$ and $\mathrm{g}(\mathrm{x}, \mathrm{t})$ are given functions. According to the variational iteration method, we construct a correction functionals as follows [18]:

$$
\begin{aligned}
& U_{n+1}(x, t)=U_{n}(x, t)+\int_{0}^{t} \lambda_{1}(\tau)\left\{L_{1} U_{n}(x, \tau)+N_{1}\left(\bar{U}_{n}(x, \tau), \bar{V}_{n}(x, \tau)\right)-f(x, \tau)\right\} d \tau \\
& V_{n+1}(x, t)=V_{n}(x, t)+\int_{0}^{t} \lambda_{2}(\tau)\left\{L_{2} U_{n}(x, \tau)+N_{2}\left(\bar{U}_{n}(x, \tau), \bar{V}_{n}(x, \tau)\right)-g(x, \tau)\right\} d \tau
\end{aligned}
$$

where $\lambda_{1}$ and $\lambda_{2}$ are general Lagrange multipliers, which can be identified, optimally, via a variational theory [2]. The second term on the right-hand side of equations (2) and (3) is called the correction and the subscript ' $n$ ' denotes the $\mathrm{n}^{\text {th }}$ order approximation. $\bar{U}_{n}$ and $\bar{V}_{n}$ are considered as a restricted variation, one can assume that the above correction functionals are stationary i.e. $\delta \bar{U}_{n}=0$ and $\delta \bar{V}_{n}=0$, then, the Lagrange multipliers can be identified.

Now we apply the Homotopy Perturbation method to the correction functional in equations (2) and (3) above. With the introduction of the power series into the correction functionals, we have the following: 


$$
\begin{aligned}
& U_{0}+P U_{1}+P^{2} U_{2}+\ldots=U_{0}-P \int_{0}^{t} \lambda_{1}(\tau)\left\{L_{1}\left(U_{0}+P U_{1}+P^{2} U_{2}+\ldots\right)\right. \\
& \left.\quad+N_{1}\left(\left(\bar{U}_{0}+P \bar{U}_{1}+P^{2} \bar{U}_{2}+\ldots .\right)+\left(\bar{V}_{0}+P \bar{V}_{1}+P^{2} \bar{V}_{2}+\ldots . .\right)\right)\right\} d \tau \\
& \quad-\int_{0}^{t} \lambda_{1}(\tau) f(x, \tau) d \tau \\
& V_{0}+P V_{1}+P^{2} V_{2}+\ldots=V_{0}-P \int_{0}^{t} \lambda_{2}(\tau)\left\{L_{2}\left(U_{0}+P U_{1}+\ldots\right)\right. \\
& \left.+N_{2}\left(\left(\bar{U}_{0}+P \bar{U}_{1}+\ldots\right)\left(\bar{V}_{0}+P \bar{V}_{1}+\ldots . .\right)\right)\right\} d \tau-\int_{0}^{t} \lambda_{1}(\tau) f(x, \tau) d \tau
\end{aligned}
$$

which can be expressed as:

$$
\begin{gathered}
\sum_{n=0}^{\infty} P^{n} U_{n}=U_{0}(x, t)+P \int_{0}^{t} \lambda_{1}(\tau)\left[L_{1} \sum_{n=0}^{\infty} P^{n} U_{n}+N_{1} \cdot\left(\sum_{n=0}^{\infty} P^{n} \bar{U}_{n}, \sum_{n=0}^{\infty} P^{n} \bar{V}_{n}\right) d \tau\right. \\
-\int_{0}^{t} \lambda_{1}(\tau) f(x, \tau) d \tau \\
\sum_{n=0}^{\infty} P^{n} V_{n}=V_{0}(x, t)+P \int_{0}^{t} \lambda_{2}(\tau)\left[L_{2} \sum_{n=0}^{\infty} P^{n} U_{n}+N_{2} \cdot\left(\sum_{n=0}^{\infty} P^{n} \bar{U}_{n}, \sum_{n=0}^{\infty} P^{n} \bar{V}_{n}\right) d \tau-\right. \\
-\int_{0}^{t} \lambda_{2}(\tau) g(x, \tau) d \tau
\end{gathered}
$$

Hence, equations (6) and (7) represent the New Variational Homotopy Perturbation method for systems of partial differential equations.

The comparison of the coefficients of like powers of $\mathrm{p}$ gives solution of various orders. This implies:

$$
\begin{gathered}
P^{0}: U_{0}=U_{0}(x, t)-\int_{0}^{t} \lambda_{1}(\tau) f(x, \tau) d \tau \\
V_{0}=V_{0}(x, t)-\int_{0}^{t} \lambda_{2}(\tau) g(x, \tau) d \tau \\
P^{1}: U_{1}=\int_{0}^{t} \lambda_{1}(\tau)\left(L_{1} U_{0}+N_{1}\left(\bar{U}_{0}, \bar{V}_{0}\right)\right) d \tau \\
V_{1}=\int_{0}^{t} \lambda_{2}(\tau)\left(L_{2} U_{0}+N_{2}\left(\bar{U}_{0}, \bar{V}_{0}\right)\right) d \tau \\
P^{2}: U_{2}=\int_{0}^{t} \lambda_{1}(\tau)\left(L_{1} U_{1}+N_{1}\left(\bar{U}_{1}, \bar{V}_{1}\right)\right) d \tau \\
: V_{2}=\int_{0}^{t} \lambda_{2}(\tau)\left(L_{2} U_{2}+N_{2}\left(\bar{U}_{1}, \bar{V}_{1}\right)\right) d \tau \\
P^{n}: U_{n}=\int_{0}^{t} \lambda_{1}(\tau)\left(L_{1} U_{n-1}+N_{1}\left(\bar{U}_{n-1}, \bar{V}_{n-1}\right)\right) d \tau \\
: V_{n}=\int_{0}^{t} \lambda_{2}(\tau)\left(L_{2} U_{n-1}+N_{2}\left(\bar{U}_{n-1}, \bar{V}_{n-1}\right)\right) d \tau
\end{gathered}
$$

Therefore, the series solutions are given as: 
$U(x, t)=U_{0}+U_{1}+U_{2}+\ldots \ldots . . U_{n}$
$V(x, t)=V_{0}+V_{1}+V_{2}+\ldots \ldots . . V_{n}$

Hence,

$$
\begin{aligned}
& U(x, t)=U_{0}(x, t)+\int_{0}^{t} \lambda_{1}(\tau)\left\{L_{1} U_{0}+N_{1}\left(\bar{U}_{0}, \bar{V}_{0}\right\} d \tau+\right. \\
& \quad \int_{0}^{t} \lambda_{1}(\tau)\left\{L_{1} U_{1}+N_{1}\left(\bar{U}_{1}, \bar{V}_{1}\right\} d \tau+\ldots \ldots \int_{0}^{t} \lambda_{1}(\tau)\left\{L_{1} U_{n-1}+N_{1}\left(\bar{U}_{n-1}, \bar{V}_{n-1}\right)\right\} d \tau\right. \\
& -\int_{0}^{t} \lambda_{1}(\tau) f(x, \tau) d \tau
\end{aligned}
$$

and

$$
\begin{aligned}
& V(x, t)=V_{0}(x, t)+\int_{0}^{t} \lambda_{2}(\tau)\left\{L_{2} U_{0}+N_{2}\left(\bar{U}_{0}, \bar{V}_{0}\right\} d \tau+\right. \\
& \quad \int_{0}^{t} \lambda_{2}(\tau)\left\{L_{2} U_{1}+N_{2}\left(\bar{U}_{1}, \bar{V}_{1}\right\} d \tau+\ldots . . \int_{0}^{t} \lambda_{2}(\tau)\left\{L_{2} U_{n-1}+N_{2}\left(\bar{U}_{n-1}, \bar{V}_{n-1}\right)\right\} d \tau\right. \\
& -\int_{0}^{t} \lambda_{2}(\tau) g(x, \tau) d \tau
\end{aligned}
$$

Example 1:

\section{Numerical Examples}

We consider the model equations for the coupled Schrodinger-kdv equation given by Doosthoseini and Shahmohamadi (2010) [8]:

$\left.\begin{array}{l}U_{t}=U_{x x}+U W \\ U_{t}=6 W W_{x}+W_{x x}=\left(|U|^{2}\right)_{x}\end{array}\right\}$

Where $i=\sqrt{-1}$

By using $U=u+i v$, one can separate equation (12) into real and imaginary parts demonstrated by Doosthoseini and Shahmohamadi (2010). Therefore, one can get a $(1+1)$ - dimensional tripled system in the following form:

$$
\left.\begin{array}{l}
U_{t}=V_{x x}+V W=0 \\
V_{t}=U_{x x}+U W=0 \\
W_{t}=6 W W_{x}+W_{x x}-2 U U_{x}-2 V V_{x}=0
\end{array}\right\}
$$

We construct a correction functional as follows:

$$
\begin{aligned}
U_{n+1}(x, t)=U_{n}(x, t)+\int_{0}^{t} \lambda_{1}\left[\frac{\partial U_{n}(x, \tau)}{\partial \tau}-\frac{\partial^{2} \bar{V}_{n}}{\partial x^{2}}-\bar{V}_{n}(x, t) \bar{W}_{n}(x, t)\right] d \tau \\
V_{n+1}(x, t)=V_{n}(x, t)+\int_{0}^{t} \lambda_{2}\left[\frac{\partial V_{n}(x, \tau)}{\partial \tau}-\frac{\partial^{2} \bar{U}_{n}}{\partial x^{2}}-\bar{U}_{n}(x, t) \bar{W}_{n}(x, t)\right] d \tau \\
W_{n+1}(x, t)=W_{n}(x, t)+\int_{0}^{t} \lambda_{3}\left[\frac{\partial W_{n}(x, \tau)}{\partial \tau}+6 W_{n}(x, t) \frac{\partial \bar{W}_{n}(x, t)}{\partial x^{2}}-\frac{\partial^{3} \bar{W}_{n}(x, t)}{\partial x^{3}}\right. \\
\left.-2 U_{n}(x, t) \frac{\partial \bar{U}_{n}(x, t)}{\partial x}-2 V_{n}(x, t) \frac{\partial \bar{V}_{n}(x, t)}{\partial x}\right] d \tau
\end{aligned}
$$


where $\lambda_{1}, \lambda_{2}$ and $\lambda_{3}$ are general Lagrange multipliers.

Its stationary conditions can be obtained as follows:

$$
\begin{aligned}
& 1+\left.\lambda_{1}(\tau)\right|_{\tau=1}, \lambda^{1}{ }_{1}(\tau)=0 \\
& 1+\left.\lambda_{2}(\tau)\right|_{\tau=1}, \lambda^{1}{ }_{2}(\tau)=0 \\
& 1+\left.\lambda_{3}(\tau)\right|_{\tau=1}, \lambda^{1}{ }_{3}(\tau)=0
\end{aligned}
$$

Hence $\lambda_{1}=\lambda_{2}=\lambda_{3}=-1$ and the correction functional becomes

$$
\begin{aligned}
& U_{n+1}=U_{n}-\int_{0}^{t}\left(\frac{\partial U_{n}}{\partial \tau}-\frac{\partial^{2} V_{n}}{\partial x^{2}}-V_{n} W_{n}\right) d \tau \\
& V_{n+1}=V_{n}-\int_{0}^{t}\left(\frac{\partial V_{n}}{\partial \tau}-\frac{\partial^{2} U_{n}}{\partial x^{2}}-U_{n} W_{n}\right) d \tau \\
& W_{n+1}=W_{n}-\int_{0}^{t}\left(\frac{\partial W_{n}}{\partial \tau}-6 W_{n} \frac{\partial^{2} W_{n}}{\partial x^{2}}-\frac{\partial^{3} W_{n}}{\partial x^{3}}-2 U_{n} \frac{\partial U_{n}}{\partial x^{3}}-2 V_{n} \frac{\partial V_{n}}{\partial x}\right) d \tau
\end{aligned}
$$

Applying Homotopy Perturbation to equations (18), (19) and (20), we have:

For equation (18)

$$
\begin{aligned}
U_{0}+P U_{1}+P^{2} U_{2}= & U_{0}-\int_{0}^{t} P\left\{\left(\frac{\partial U_{0}}{\partial \tau}+P \frac{\partial U_{1}}{\partial \tau}+\ldots\right)-\left(\frac{\partial^{2} V_{0}}{\partial x}+P \frac{\partial V_{1}}{\partial x}+\ldots\right)\right. \\
& \left.-\left(V_{0}+P V_{1}+\ldots\right)\left(W_{0}+P W_{1}+\ldots .\right)\right\} d \tau
\end{aligned}
$$

$P^{0}: U_{0}=\cos x$

$P^{1}: U_{0}=+t\left[\frac{\partial \cos x}{\partial \tau}-\frac{\partial^{2} \sin x}{\partial \tau}-\sin x(3 / 4)\right]$

$=-(t / 4) \sin x$

For equation (19)

$$
\begin{gathered}
V_{0}+P V_{1}+P^{2} V_{2}=V_{0}-\int_{0}^{t} P\left\{\left(\frac{\partial U V_{0}}{\partial \tau}+P \frac{\partial V_{1}}{\partial \tau}+\ldots\right)-\left(\frac{\partial^{2} U_{0}}{\partial x^{2}}+P \frac{\partial U_{1}}{\partial x^{2}}+\ldots\right)\right. \\
\left.-\left(U_{0}+P U_{1}+\ldots\right)\left(W_{0}+P W_{1}+\ldots .\right)\right\} d \tau
\end{gathered}
$$

Comparing the coefficients of like powers of $\mathrm{p}$ and using the initial conditions of above:

$$
\begin{aligned}
& P^{0}: V_{0}=\sin x \\
& P^{1}: V_{1}=-t(0-\cos x+3 / 4 \cos x) \\
& \quad=t / 4 \cos x
\end{aligned}
$$

For equation (20)

$$
\begin{aligned}
W_{0}+P W_{1}+\ldots & =W_{0}-P \int_{0}^{t}\left\{\left(\frac{\partial W_{0}}{\partial \tau}+P \frac{\partial W_{1}}{\partial \tau}+\ldots\right)+6\left(W_{0}+P W_{1}+\ldots\right)\right. \\
( & \left.\frac{\partial^{2} W_{0}}{\partial x^{2}}+P \frac{\partial^{2} W_{1}}{\partial x^{2}}+\ldots\right)-\left(\frac{\partial^{3} W_{0}}{\partial x^{3}}+P \frac{\partial^{3} W_{1}}{\partial x^{3}}+\ldots\right) \\
& -2\left(U_{0}+P U_{1}+\ldots\right)\left(\frac{\partial U_{0}}{\partial x}+P \frac{\partial W_{1}}{\partial x}+\ldots\right) \\
& \left.-2\left(V_{0}+P V_{1}+\ldots .\right)\left(\frac{\partial V_{0}}{\partial x}+P \frac{\partial V_{1}}{\partial x}+\ldots\right)\right\} d \tau
\end{aligned}
$$


Comparing the coefficients of like power of $\mathrm{p}$ :

$$
\begin{aligned}
& P^{0}: W_{0}=3 / 4 \\
& P^{1}: W_{1}=-t(0-6(3 / 4)(0)+(0)-2 \cos x(-\sin x)-2 \sin x \cos x) \\
& =0
\end{aligned}
$$

Following the same procedure, we have

$$
\begin{aligned}
P^{2}: U_{2} & =-t\left(\frac{\partial U_{1}}{\partial \tau}-\frac{\partial^{2} U_{2}}{\partial x^{2}}-V_{1} W_{1}\right) \\
= & -\frac{t}{4} \sin x-\frac{t^{2}}{4} \cos x \\
P^{2}: V_{2} & =-t\left(\frac{\partial V_{1}}{\partial \tau}-\frac{\partial^{2} V_{2}}{\partial x^{2}}-U_{1} W_{1}\right) \\
& =-\frac{t}{4} \cos x-\frac{t^{2}}{4} \sin x \\
P^{2}: W_{2} & =-t\left(\frac{\partial W_{1}}{\partial \tau}-\frac{6 W_{1} \partial^{2} W_{1}}{\partial x^{2}}-\frac{\partial^{3} W_{1}}{\partial x^{3}}-\frac{2 U_{1} \partial^{2} U_{1}}{\partial x}-\frac{2 V_{1} \partial V_{1}}{\partial x}\right) \\
=0 &
\end{aligned}
$$

\begin{tabular}{|c|c|c|c|c|c|}
\hline & & Table 1 & imple 1 for & & \\
\hline $\mathrm{X}$ & EXACT & VIM/HPM & ERROR & NVHPM & ERROR \\
\hline-0.5 & 0.999941 & 1.006683 & $6.742 \times 10^{-3}$ & 1.062460 & $6.2519 \times 10^{-2}$ \\
\hline-0.4 & 0.999962 & 1.004277 & $4.315 \times 10^{-3}$ & 1.039975 & $4.0013 \times 10^{-2}$ \\
\hline-0.3 & 0.999979 & 1.002406 & $2.427 \times 10^{-3}$ & 1.022486 & $2.2507 \times 10^{-2}$ \\
\hline-0.2 & 0.999990 & 1.001069 & $1.079 \times 10^{-3}$ & 1.009994 & $1.0004 \times 10^{-2}$ \\
\hline-0.1 & 0.999998 & 1.000267 & $2.690 \times 10^{-4}$ & 1.002498 & $2.5000 \times 10^{-3}$ \\
\hline 0 & 1.000000 & 1.000000 & 0.000000 & 1.000000 & 0.000000 \\
\hline 0.1 & 0.999998 & 0.999642 & $3.560 \times 10^{-4}$ & 0.997498 & $2.5000 \times 10^{-3}$ \\
\hline 0.2 & 0.999990 & 0.998569 & $1.421 \times 10^{-3}$ & 0.989994 & $9.9960 \times 10^{-3}$ \\
\hline 0.3 & 0.999979 & 0.996781 & $3.198 \times 10^{-3}$ & 0.977487 & $2.2494 \times 10^{-2}$ \\
\hline 0.4 & 0.999962 & 0.994277 & $5.685 \times 10^{-3}$ & 0.959977 & $3.9985 \times 10^{-2}$ \\
\hline 0.5 & 0.999941 & 0.991059 & $8.882 \times 10^{-3}$ & 0.937464 & $6.2477 \times 10^{-2}$ \\
\hline
\end{tabular}

Therefore the solutions are:

$$
\begin{aligned}
& U(x, t)=\cos x-\frac{t}{4} \sin x+\frac{t}{4} \sin x-\frac{t^{2}}{4} \cos x=\cos x\left(1-\frac{t^{2}}{4}\right) \\
& V(x, t)=\sin x-\frac{t}{4} \cos x+\frac{t}{4} \cos x-\frac{t^{2}}{4} \sin x=\sin x\left(1-\frac{t^{2}}{4}\right) \\
& W(x, t)=\frac{3}{4}+0+0=\frac{3}{4}
\end{aligned}
$$


Table 2 for example 1 for $\mathrm{V}$ :

\begin{tabular}{|c|c|c|c|c|c|}
\hline$X$ & EXACT & VIM/HPM & ERROR & NVHPM & ERROR \\
\hline 0.5 & 0.010908 & 0.133790 & $\begin{array}{l}1.22882 \times 10 \\
-1\end{array}$ & 0.009272 & $1.636 \times 10^{-3}$ \\
\hline 0.4 & 0.008727 & 0.107014 & $9.8287 \times 10^{-2}$ & 0.007261 & $1.466 \times 10^{-3}$ \\
\hline .3 & 0.006545 & 0.080250 & $7.3705 \times 10^{-2}$ & 0.005354 & $1.191 \times 10^{-3}$ \\
\hline 0.2 & 0.004363 & 0.053495 & $4.9132 \times 10^{-2}$ & 0.003526 & $8.37 \times 10^{-4}$ \\
\hline 0.1 & 0.002182 & 0.026746 & $2.4564 \times 10^{-2}$ & 0.001750 & $4.32 \times 10^{-4}$ \\
\hline 0 & 0.000000 & 0.000000 & 0.000000 & 0.000000 & 0.000000 \\
\hline 0.1 & 0.002182 & 0.026745 & $2.4563 \times 10^{-2}$ & 0.001741 & $4.42 \times 10^{-4}$ \\
\hline 0.2 & 0.004363 & 0.053486 & $4.9123 \times 10^{-2}$ & 0.003456 & $9.06 \times 10^{-4}$ \\
\hline 0.3 & 0.006545 & 0.080220 & $7.3675 \times 10^{-2}$ & 0.005118 & $1.427 \times 10^{-3}$ \\
\hline 0.4 & 0.008727 & 0.106944 & $9.8217 \times 10^{-2}$ & 0.006702 & $2.025 \times 10^{-3}$ \\
\hline 0.5 & 0.010908 & 0.133654 & $\begin{array}{l}1.22746 \times 10 \\
-1\end{array}$ & 0.008181 & $2.727 \times 10^{-3}$ \\
\hline
\end{tabular}

Table 3 for Example 1:

$\begin{array}{llllll}\text { X } & \text { EXACT } & \text { VIM/HPM } & \text { ERROR } & \text { NVHPM } & \text { ERROR } \\ -0.5 & 0.750000 & 0.750000 & 0.000000 & 0.750000 & 0.000000 \\ -0.4 & 0.750000 & 0.750000 & 0.000000 & 0.750000 & 0.000000 \\ -0.3 & 0.750000 & 0.750000 & 0.000000 & 0.750000 & 0.000000 \\ -0.2 & 0.750000 & 0.750000 & 0.000000 & 0.750000 & 0.000000 \\ -0.1 & 0.750000 & 0.750000 & 0.000000 & 0.750000 & 0.000000 \\ 0 & 0.750000 & 0.750000 & 0.000000 & 0.750000 & 0.000000 \\ 0.1 & 0.750000 & 0.750000 & 0.000000 & 0.750000 & 0.000000 \\ 0.2 & 0.750000 & 0.750000 & 0.000000 & 0.750000 & 0.000000 \\ 0.3 & 0.750000 & 0.750000 & 0.000000 & 0.750000 & 0.000000 \\ 0.4 & 0.750000 & 0.750000 & 0.000000 & 0.750000 & 0.000000 \\ 0.5 & 0.750000 & 0.750000 & 0.000000 & 0.750000 & 0.000000\end{array}$

Example 2:

We consider a coupled system of nonlinear physical equations given by Abdoul et al (2009) [2]:

$$
\begin{aligned}
& \frac{\partial U(x, t)}{\partial t}=U\left(1-U^{2}-V\right)+U_{x x}, t>0 \\
& \frac{\partial V(x, t)}{\partial t}=V(1-U-V)+V_{x x},
\end{aligned}
$$

With initial conditions

$$
\begin{aligned}
& U(x, 0)=\frac{e^{k x}}{\left[1+e^{k x}\right]} \\
& V(x, 0)=\frac{1+(3 / 4) e^{k x}}{\left[1+e^{k x}\right]^{2}},
\end{aligned}
$$

and the exact solutions are

$$
\begin{aligned}
& U(x, t)=\frac{e^{k(x+(t)}}{\left.1+e^{k(x+(t)}\right]} \\
& V(x, t)=\frac{1+(3 / 4) e^{k(x+(t)}}{\left[1+e^{k(x+(t)}\right]^{2}},
\end{aligned}
$$


The correction functionals are given as

$$
\begin{gathered}
U_{n+1}=U_{n}(x, t)-\int_{0}^{t}\left[\frac{\partial U_{n}(x, \tau)}{\delta \tau}-\frac{\partial^{2} U_{n}(x, t)}{\delta x^{2}}-U_{n}(x, t)+U^{3}{ }_{n}(x, t)\right. \\
\left.-U_{n}(x, t) V_{n}(x, t)\right] d \tau \\
V_{n+1}(x, t)=V_{n}(x, t)-\int_{0}^{t}\left[\frac{\partial V_{n}(x, \tau)}{\delta \tau}-\frac{\partial^{2} V_{n}(x, t)}{\delta x^{2}}-V_{n}(x, t)+V_{n}^{2}(x, t)\right. \\
\left.-U_{n}(x, t) V_{n}(x, t)\right] d \tau
\end{gathered}
$$

With $\lambda=-1$

Applying the Homotopy Perturbation method to equations (30) \& (31), we have

$$
\begin{aligned}
U_{0} & +P U_{1}+P^{2} U_{2}+\ldots=U_{0}-P \int_{0}^{t}\left[\frac{\partial}{\delta \tau}\left(U_{0}+P U_{1}+\ldots\right)-\frac{\partial^{2}}{\delta x^{2}}\left(U_{0}+P U_{1}+\ldots\right)\right. \\
& \left.-\left(U_{0}+P U_{1}+\ldots\right)+\left(U_{0}+P U_{1}+\ldots\right)^{2}+\left(U_{0}+P U_{1}+\ldots\right)\left(V_{0}+P V_{1}+\ldots\right)\right] d \tau
\end{aligned}
$$

and

$$
\begin{aligned}
V_{0}+ & P V_{1}+P^{2} V_{2}+\ldots=V_{0}-P \int_{0}^{t}\left[\frac{\partial}{\delta \tau}\left(V_{0}+P V_{1}+\ldots\right)-\frac{\partial^{2}}{\delta x^{2}}\left(V_{0}+P V_{1}+\ldots\right)\right. \\
& \left.-\left(V_{0}+P V_{1}+\ldots\right)+\left(V_{0}+P V_{1}+\ldots\right)^{2}+\left(U_{0}+P U_{1}+\ldots\right)\left(V_{0}+P V_{1}+\ldots\right)\right] d \tau
\end{aligned}
$$

Comparing the coefficients of like powers of $\mathrm{p}$, we have

$$
\begin{aligned}
P^{0}: U_{0} & =\frac{e^{k x}}{\left[1+e^{k x}\right]} \\
: V_{0} & =\frac{1+(3 / 4) e^{k x}}{\left[1+e^{k x}\right]^{2}} \\
P^{0}: U_{1} & =\frac{4 k^{2} e^{k x} t-4 k^{2} e^{3 k x} t+5 e^{2 k x} t+5 e^{3 k x} t}{4\left[1+e^{k x}\right]^{4}} \\
: V_{1} & =\frac{16 k e^{k x} t-32 e^{k x} t+21 e^{2 k x} t+16 e^{3 k x} t}{16\left[1+e^{k x}\right]^{4}}
\end{aligned}
$$

Therefore, the series solutions are

$$
\begin{aligned}
U & =U_{0}+U_{1}+\ldots . . \\
& =\frac{\left[4 e^{k x}\left(1+e^{k x}\right)^{3}+\left(4 k^{2} e^{k x} t-4 k^{2} e^{3 k x} t+5 e^{2 k x} t+5 e^{3 k x} t\right)\right]}{4\left(1+e^{k x}\right)^{4}}
\end{aligned}
$$

and

$$
\begin{aligned}
V & =V_{0}+V_{1}+\ldots . . \\
& =\frac{\left[16+44 e^{k x} t+40 e^{k x} t+12 e^{3 k x} t+16 k e^{k x} t+32 e^{k x} t+21 e^{2 k x} t+16^{3 k x} t\right]}{16\left(1+e^{k x}\right)^{4}}
\end{aligned}
$$




\begin{tabular}{llllll}
\hline & \multicolumn{7}{c}{ TABLE 1 FOR EXAMPLE 2 FOR U: } \\
$\mathrm{X}$ & EXACT & VIM/HPM & ERROR & NVHPM & \multicolumn{2}{c}{ ERROR } \\
0.1 & 0.54983 & 0.27629 & $2.735 \times 10^{-1}$ & 0.540098 & $9.732 \times 10^{-3}$ \\
0.2 & 0.59869 & 0.30535 & $2.933 \times 10^{-1}$ & 0.68032 & $8.163 \times 10^{-2}$ \\
0.3 & 0.64566 & 0.33746 & $3.082 \times 10^{-1}$ & 0.69946 & $5.380 \times 10^{-2}$ \\
0.4 & 0.68997 & 0.37296 & $3.170 \times 10^{-1}$ & 0.71815 & $2.818 \times 10^{-2}$ \\
0.5 & 0.73106 & 0.41218 & $3.189 \times 10^{-1}$ & 0.73637 & $5.311 \times 10^{-2}$
\end{tabular}

\begin{tabular}{|c|c|c|c|c|c|}
\hline & & TABLE & XAMPLE 2 & & \\
\hline $\mathrm{X}$ & EXACT & VIM/HPM & ERROR & NVHPM & ERROR \\
\hline 0.1 & 0.17479 & 0.003182 & $1.716 \times 10^{-1}$ & 0.161797 & $1.299 \times 10^{-2}$ \\
\hline 0.2 & 0.13695 & 0.002567 & $1.344 \times 10^{-1}$ & 0.135013 & $1.937 \times 10^{-3}$ \\
\hline 0.3 & 0.10529 & 0.002050 & $1.032 \times 10^{-1}$ & 0.111854 & $6.564 \times 10^{-3}$ \\
\hline 0.4 & 0.07954 & 0.001621 & $7.792 \times 10^{-2}$ & 0.092062 & $1.252 \times 10^{-4}$ \\
\hline 0.5 & 0.05911 & 0.001270 & $5.784 \times 10^{-2}$ & 0.075330 & $1.622 \times 10^{-4}$ \\
\hline
\end{tabular}

\section{Conclusion}

In this paper, New Variational Homotopy Perturbation Method has been successfully applied to find the solutions of system of partial differential equations and the results obtained were compared with the two convectional variational iteration and Homotopy Perturbation Method. It can be concluded that the NVHPM is very power and efficient technique for finding approximation solutions for wide classes of problems. It is worth mentioning that the Method is computational cost friendly.

\section{References:}

[1] Abdou, M.A. "New Applications of He's Homotopy Perturbation Method for Nonlinear Differential Difference Equations", Physica Scripta, 81(2010)015003(8pp).

[2] Abdoul R. Ghotbi etal, "Application of Variational Iteration Method to Coupled System of Nonlinear Partial Differential Physical Equations", Africa Journal of Maths and Computer Science Research vol.2(4),pp.063-068, may,2009.

[3] Adomian, G., "Solving Frontier Problems of Physics: The Decomposition Method, kluwer Academic press,(1994).

[4] Aminikhah H. and Salahi M., "A new HPM for Integral Equations", Applications and Applied Maths, vol.4, No.1 (June 2009) pp.122-133.

[5] Barari A., Ghotbi A.R. and Ganji D.D, 'Variational Iteration Method and Homotopy-perturbation Method for Solving Different Types of Wave Equations"',Journal of Applied Science 8(1):120-126,2008.

[6] Batiha B., Noorani M.S.M. and Hashim I, "Numerical solutions of the nonlinear integro-differential equations", int. J. open problems compt. Math., vol. 1, No. 1, June 2008.

[7] Biazar J. and Ebrahimi H., "Variational Iteration Method for Fredholm Integral Equations of the Second Kind", Iranian Journal of Optimization 1(2009), Pp 13-23.

[8] Doosthoseini A. and Shahmohamadi H., "Variational Iteration Method for Solving Coupled schrodinger-Kdv Equation", Applied Mathematical Sciences, vol.4,2010,no.17,823-837.

[9] He J.H.,"A Coupling Method Of A Homotopy Technique And A Perturbation Technique For Nonlinear Problems,” International Journal of Non-Linear Mechanics, vol. 35, no. 1, pp. 37-43, 2000.

[10] He J.H., "Variational Iteration Method-A Kind Of Nonlinear Analytical Technique: Some Examples," International Journal of Non-Linear Mechanics, vol. 34, no. 4, pp. 699-708, 1999.

[11] He J.H.,"Variational Iteration Method For Autonomous Ordinary Differential Systems," Applied Mathematics and Computation, vol. 114, no. 2-3, pp. 115-123, 2000.

[12] He J.H., "Some Asymptotic Methods For Strongly Nonlinear Equations," International Journal of Modern Physics B, vol. 20, no. 10 , pp. 1141-1199, 2006.

[13] He J.H., "Variational Iteration Method-Some Recent Results And New Interpretations," Journal of Computational and Applied Mathematics, vol. 207, no. 1, pp. 3-17, 2007.

[14] He J.H., "The Variational Iteration Method for Eighth-Order Initial-Boundary Value Problems," Physica Scripta, vol. 76, no. 6, pp. $680-682,2007$.

[15] Hesameddini E. and Latifizadeh H., "New Approach to Mixture of the Adomian Decomposition Homotopy Perturbation for Approximate and Analytical Solution of Integral Equations Fractional Differential Equations", Applied Mathematical Sciences, vol.3,2009.no.572797-2809.

[16] Inokuti M., Sekine H., and Mura T., "General Use of the Lagrange Multiplier in Nonlinear Mathematical Physics," in Variational Method in the Mechanics of Solids, S. Nemat-Naseer, Ed., pp. 156-162, Pergamon Press, New York, NY, USA, 1978.

[17] Noor M.A. and Mohyud-Din S.T. "Modified Variational Iteration Method for Goursat and Laplace problems", World Applied Sciences Journal 4(4):487-498, 2008.

[18] Noor M.A. and Mohyud-Din S.T. "Variational Iteration Method for Fifth-order Boundary Value Problems Using HE's Polynomials", Mathematical Problems in Eng. Vol.2008, article ID954794. 